\title{
Influence of Different LED Light Colour Temperatures on the Preference Behaviour of Weaned Piglets
}

\author{
Sven Götz ${ }^{1,2, *(\mathbb{D}}$, Camille M. C. Raoult ${ }^{1}{ }^{\mathbb{D}}$, Klaus Reiter ${ }^{2}$, Monika Wensch-Dorendorf ${ }^{3}{ }^{\mathbb{D}}$, \\ Daniel Werner ${ }^{4,5}$ and Eberhard von Borell ${ }^{1, *}$ \\ 1 Institute of Agricultural and Food Sciences, Department of Animal Husbandry and Ecology, \\ Martin-Luther-University Halle-Wittenberg, Theodor-Lieser-Str. 11, 06120 Halle (Saale), Germany; \\ camille.raoult@landw.uni-halle.de \\ 2 Bavarian State Office for Agriculture, Institute for Agricultural Engineering and Animal Husbandry, \\ Prof.-Dürrwaechter-Platz 5, 85586 Poing-Grub, Germany; klaus.reiter@lfl.bayern.de \\ 3 Institute of Agricultural and Nutritional Sciences, Department of Biometrics and Agricultural Informatics, \\ Martin-Luther-University Halle-Wittenberg, Karl-Freiherr-von-Fritsch-Str. 4, 06120 Halle (Saale), Germany; \\ monika.dorendorf@landw.uni-halle.de \\ 4 Centre for Agriculture Haus Düsse, Ostinghausen, 59505 Bad Sassendorf, Germany; \\ daniel.werner@lwk.nrw.de \\ 5 Institute for Technical Energy Systems, University of Applied Sciences Bielefeld, Interaktion 1, \\ 33619 Bielefeld, Germany \\ * Correspondence: sven.goetz@lfl.bayern.de (S.G.); eberhard.vonborell@landw.uni-halle.de (E.v.B.); \\ Tel.: +49-345-5522-336 (S.G.); +49-345-5522-330 (E.v.B.)
}

Received: 9 November 2020; Accepted: 30 November 2020; Published: 2 December 2020

\begin{abstract}
This study investigates the effect of different LED lighting colour temperatures on the preference behaviour of 4-week-old weaned piglets. A total of 32 piglets were housed in two replications in an experimental pen area with four identically equipped pen compartments connected two by two. Each pen unit offered a compartment set to a colour temperature of 3000 kelvin and another set to 6500 kelvin, at 80 lux during the day. Each piglet could freely choose between the two compartments by using a passageway. Over a period of five weeks, the behaviours "lying", "eating" and "activity" were video recorded for $72 \mathrm{~h}$ during the 1st, 3rd and 5th week of the experiment. The location of the piglet in the pen and its behaviour were determined by using time sampling. In the first week, the piglets preferred the colour temperature of $3000 \mathrm{~K}$ to perform all behaviours. In the following weeks this preference decreased. Results also show that feed consumption and soiling of the pens were higher under $6500 \mathrm{~K}$. Pigs can differentiate between the different colour temperatures and use them for different behaviours. This can be used to divide pens into functional areas in order to better suit the behavioural needs of pigs.
\end{abstract}

Keywords: colour temperature; piglets; preference behaviour; light-emitting diode (LED); pig housing

\section{Introduction}

The effect of lighting environment on pig performance and welfare has been poorly investigated and therefore clear recommendations are lacking. Currently, the German animal welfare legislation describes as uniform lighting requirements for housing pigs, an illuminance of at least 80 lux over a period of $8 \mathrm{~h} \mathrm{[1]}$, while a level of at least 40 lux over a period of $8 \mathrm{~h}$ is required in the EU Directive 2008/120/EC [2]. In Australia and New Zealand, this value is further reduced to a minimum of 20 lux over a period of $9 \mathrm{~h}[3,4]$. In the United States, on the other hand, the minimal recommended value arises to 100 lux [5]. However, none of these recommendations are based on sufficient scientific evidence. Neither are there recommendations for a maximum upper limit for the illuminance, which under 
natural conditions can reach several 10000 lux [6]. Moreover, the type and colour temperature of the light sources to be used is not described in great detail in the legal requirements. Recently, a surge of interest for light-emitting diode (LED) lights, for example in poultry housing, has been observed (e.g., in broiler chickens $[7,8]$ ). LED lights emit a complex spectrum and the different wavelengths determine the light colour. The visual system of the pig is very similar to that of humans, though a large difference exists in the way pigs see colours. Contrary to the trichromatic humans, pigs are dichromate, meaning that they have only two types of cones in their eyes [9]. Cones are specialised sensory cells in the retina of the eye allowing coloured light to be absorbed, while rods are very sensitive to light and allow for brightness vision [10]. The human eye has three types of cones that enable them to distinguish colours: an S cone (blue cone) for short wavelengths, an M cone (green cone) and an L cone (red cone), with a maximum sensitivity at $430 \mathrm{~nm}, 535 \mathrm{~nm}$ and $565 \mathrm{~nm}$, respectively [11]. In pigs, the maximum sensitivity is at $439 \mathrm{~nm}$ for the blue cone and $556 \mathrm{~nm}$ for the green cone, whereas the L-cone responsible for recording the long-wave spectra $(>650 \mathrm{~nm})$ is missing [12]. Certain light wavelengths have been reported to affect the behaviour, performance and welfare of broiler chickens $[7,8]$, mice $[13,14]$ and dairy cows [15]. However, to our knowledge, no study ever investigated the influence of different light colour temperatures on the behaviour of pigs.

The aim of this study was therefore to identify whether piglets can discriminate between the extreme white light colour temperatures of 3000 kelvin (reddish for human vision) and 6500 kelvin (bluish), by assessing how LED light colour temperatures influence their resting, feeding and activity behaviour. Pig preference for one or the other colour temperature was evaluated based on the percentage of pigs present in each colour temperature compartment. To measure the effect of the LED light colour temperature on the pigs' use of the pen, feed intake and pen compartment soiling were also recorded.

\section{Materials and Methods}

\subsection{Animals}

A total of 32 weaned piglets (Large-White $\times$ Landrace $\times$ Piétrain cross breed; 4-weeks old and $8.2 \pm 0.8 \mathrm{~kg}$ (mean $\pm \mathrm{SD})$ at their arrival; including 16 females and 16 castrated males) were examined in two successive batches in the preference test room of the Martin Luther University of Halle-Wittenberg, Germany. Animals had ad libitum access to pellet feed (Mini Start, Denkapig; FA I, Agravis, Querfurt, Germany) and water from nipple drinkers. The experiment was notified to the Saxony-Anhalt Regional Administrative Office (Veterinary Affairs) but required no permission with regard to the Animal Protection Law ( $\$ 7$, paragraph 2) since no measures inflicting pain, suffering, or injury to these animals were carried out.

\subsection{Experimental Design}

The preference test room consisted of 4 identically equipped pen compartments measuring $4.17 \mathrm{~m}^{2}$ (i.e., $1.67 \mathrm{~m} \times 2.49 \mathrm{~m}$ ) each (Figure 1). Each pen compartment consisted of half of the surface of a concrete slatted floor (with a slot width of $10 \mathrm{~mm}$ ), a heating plate (MIK International THERMO E, MIK International, Ransbach-Baumbach; Germany; $400 \mathrm{~mm} \times 600 \mathrm{~mm}$ ) and a food trough, and on the other half of the surface were plastic floor grids (MIK International Rubin, MIK International, Ransbach-Baumbach, Germany; slot width of $10 \mathrm{~mm}$ ) and a nipple drinker. Two lightings were installed above each compartment. Pen compartments were connected two by two (i.e., compartments 1 and 2, and compartments 3 and 4; referred to as pen units) and piglets could move from one compartment to the other by using a passageway (Figure 1). The passageways were $47 \mathrm{~cm}$ (high) $\times 35 \mathrm{~cm}$ (wide) large, from which three opaque black PVC strips $(55 \mathrm{~cm}$ high $\times 20 \mathrm{~cm}$ wide $\times 0.5 \mathrm{~cm}$ thick each; Thermonet Trade, Mannheim, Germany) were hanging in order to separate the two colour temperatures. 


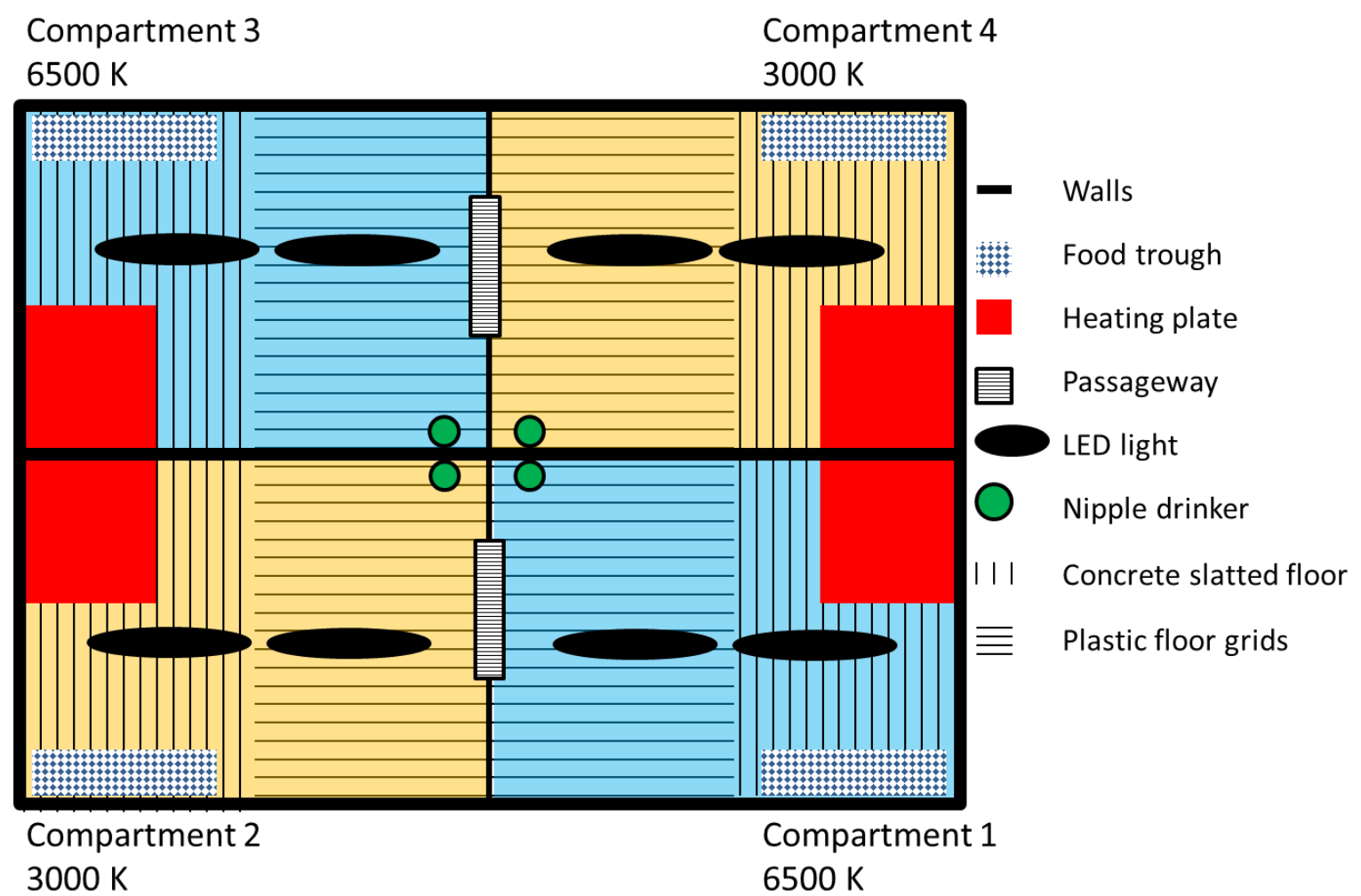

Figure 1. Design of the preference test pen compartments.

Throughout the experiment, the light phase took place from 7:00 to 19:00, with each compartment being illuminated with 80 lux. During the dark phase, from 19:00 to 7:00, the illuminance was reduced to 3 lux. In order to achieve uniform illumination of the compartments, the required light intensity was beforehand simulated with DIALux evo 9.1 software (DIAL GmbH, Lüdenscheid, Germany). Then, two custom-made LED lightings of the Schuch 161-162 type (Adolf Schuch GmbH, Worms, Germany) were installed above each compartment on a wooden beam. The lightings' illuminance within each compartment was then controlled using a Mavolux 5032B luxmeter (Gossen Foto-und Lichtmesstechnik GmbH, Nürnberg, Germany) and the installation height of the lights was adjusted. A uniform illumination of $g_{1}=0.87$ (i.e., the value corresponding to the ratio between the minimum to average light intensity) could be obtained, which is well above the minimum required value of $\mathrm{g}_{1}=0.6[16]$.

Before starting the experimental period per se, pigs had a 6-day period of acclimatization in the preference test room. This acclimatisation took place under a neutral lighting with a colour temperature of $4000 \mathrm{~K}$ and 80 lux. In each batch, 8 pigs per pen unit (i.e., 4 females and 4 castrated males) were first housed in one of the compartments for 3 days, and then in the other compartment of the unit pen for 3 more days. Closing the passageway between the two compartments of a pen unit was designed to encourage pigs to defaecate and for a resting area in each compartment. The passageway in each pen unit was then opened on the first day of the experiment. The routine stable work, including animal inspection and pen compartments' cleaning, took place daily between 8 a.m. and 10 a.m., with a rotating pen cleaning order to prevent animal habituation and behavioural changes (e.g., compartment avoidance). During the 5-weeks experiment, the neutral lighting was switched off and pigs in each pen unit could freely choose to stay in the compartment with either a 3000 kelvin LED light or a 6500 kelvin LED light (Figure 2). The $6500 \mathrm{~K}$ LED light colour temperature was switched on in pen compartments 1 and 3 for the first batch and compartments 2 and 4 for the second batch, while the 3000 K LED light was turned on in the two other compartments. 


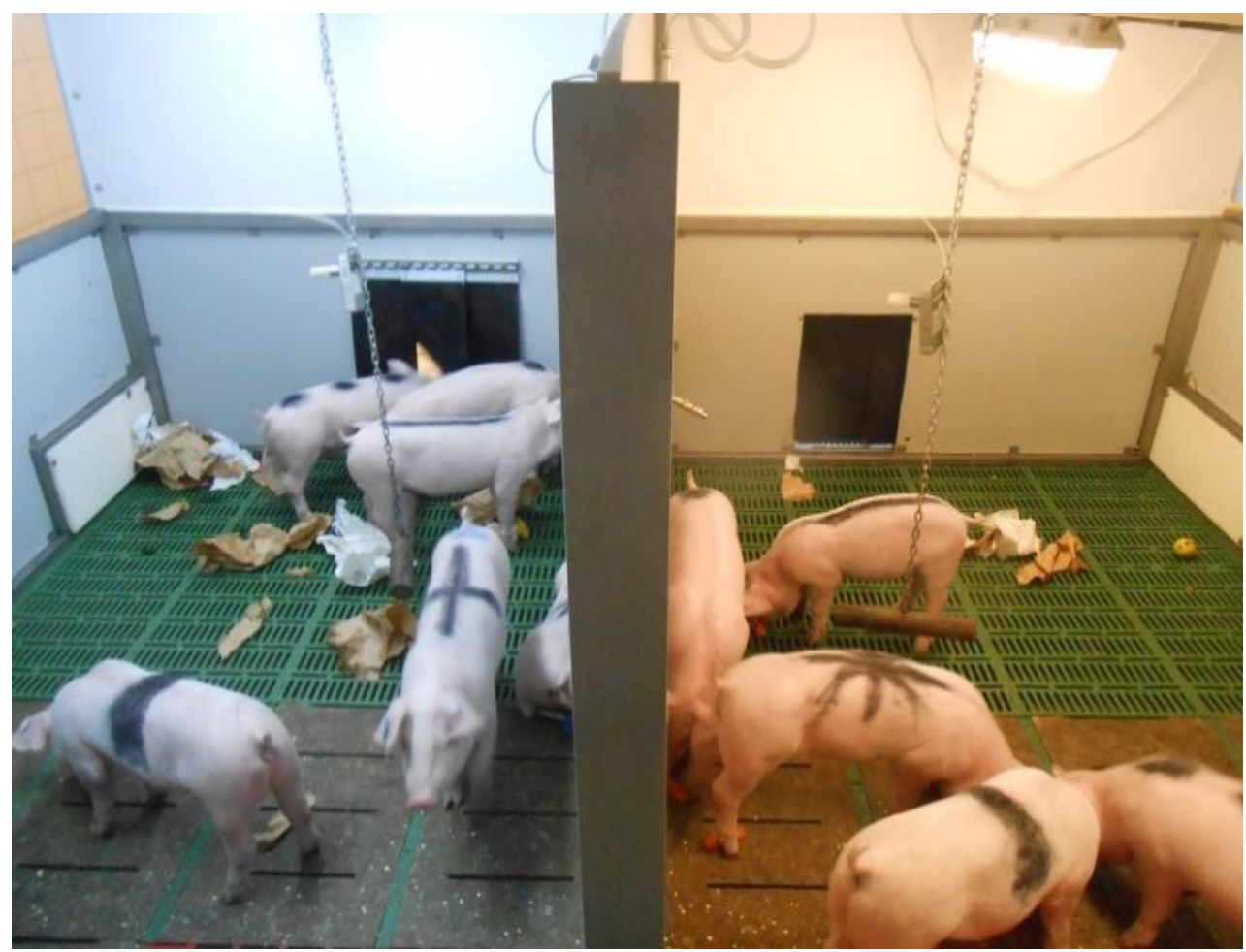

Figure 2. Pen compartments with LED light colour temperatures of $6500 \mathrm{~K}$ on the left and $3000 \mathrm{~K}$ on the right.

\subsection{Measurements}

To investigate the influence of LED light colour temperatures on the preference behaviour of weaned piglets, the cleanliness of each pen compartment, as well as the pigs' feed consumption, behaviour (i.e., lying, eating and activity) and location in the pen (i.e., colour temperature compartment) were recorded. In order to not bias the results, no behavioural observation was made during the routine stable work (that includes animal inspection and pen compartments' cleaning), i.e., between 8:00 and 10:00.

Pen compartment cleanliness was assessed daily based on the method described by the Bavarian State Office for Agriculture for measuring partially slatted floor pens' cleanliness for fattening pigs [17]. Pen compartment cleanliness was scored between 0 and 4 as follows: $0=$ not soiled, i.e., a clean pen without contamination by faeces and urine; $1=$ very lightly soiled, i.e., visible individual piles of excrements; 2 = lightly soiled, i.e., light visible soiling with excrements covering less than $25 \%$ of the floor; $3=$ moderately soiled, i.e., medium visible soiling with excrements covering more than $25 \%$ and up to $50 \%$ of the floor; and $4=$ highly soiled, i.e., high degree of pollution with excrements covering more than $50 \%$ of the floor.

To determine the pigs' feed consumption, the feed supplied daily was weighed in each pen compartment and, once a week (on Thursdays), the remaining amount of feed in each trough was weighed.

During the experiment, four video cameras (Monacor HDCAM 630, Monacor International GmbH \& Co. KG, Bremen, Germany; a 2-megapixel HD-SDI colour camera with day/night function and 2.8-12 mm varifocal lens), mounted on the ceiling above the four pen compartments, continuously recorded the animals' behaviour. Video recordings were stored on a digital recorder (EPHD 08 Everfocus, New Taipei, Taiwan). To define the pigs' behaviour and location in the pen, the video recordings of 3 alternating days (Tuesdays, Thursdays and Saturdays) within the first, third and fifth week of experiment were analysed by using a $5 \mathrm{~min}$ time sampling method. Every $5 \mathrm{~min}$ throughout the entire $24 \mathrm{~h}$ period, the video was stopped and each behaviour (i.e., lying, eating and active) and 
location (i.e., $3000 \mathrm{~K}$ or $6500 \mathrm{~K}$ compartment) was noted in a binary way (i.e., $1=$ occurring/present, $0=$ not occurring/absent) for each pig. The pig was considered as "lying" when the pig was lying down either in a sternal or lateral position; "eating", when the pig was eating, chewing or had the head above the feed trough (as no distinction could be made); and "active", when the pig was neither lying or eating (this also includes sitting pigs).

\subsection{Statistical Analyses}

Data was prepared using Microsoft Excel (Microsoft Corporation, Redmont, WA, USA) and statistical analyses were carried out using Statistical Analysis System 9.4 (SAS Institute Inc., Cary, NC, USA). To facilitate calculations and reduce the amount of data, all time sampling behavioural observations of one day were summed beforehand in 2-h sections throughout the day.

A Chi-square test was used to compare the pen compartment cleanliness score distribution under the two LED light colour temperatures (3000 K and $6500 \mathrm{~K}$ ).

To evaluate the pig preference behaviour (i.e., feed consumption, lying behaviour, eating behaviour, active behaviour and colour temperature) under different LED light colour temperatures, linear mixed models were used, with multiple comparisons being performed using the LSMEANS statement and the Tukey-Kramer adjustment for multiple comparisons. Statistical assumptions were checked using a graphical analysis of residuals focusing on the distribution and homoscedasticity of the errors of the models.

The MIXED procedure was used to examine the feed consumption. The homogeneity of variances of the data was beforehand verified with the GLM procedure using Levene's test. For the residual effects, heterogeneous residual variances were modelled (grouped according to the experimental week). Variance components were estimated using the restricted maximum likelihood (REML) method. The "feed consumption" model included the batch (factor with two levels: 1 and 2), experimental week (factor with five levels: 1-5), pen unit (factor with two levels: 1,2), compartment colour temperature (factor with two levels: $3000 \mathrm{~K}$ and $6500 \mathrm{~K}$ ) and their interactions as fixed effects. F-tests of overall significance $(p<0.05)$ were calculated in order to retain meaningful variables and interactions only, apart from the compartment colour temperature, which was inevitably kept. The final "feed consumption" model included the experimental week, compartment colour temperature and their interactions as fixed effects.

The GLIMMIX procedure was used to calculate the probability for the piglets to be under one or the other LED light colour temperatures. Proportions under this binomial distribution were logit transformed for the evaluation. Variance components were estimated using the maximum likelihood estimation method. The model included the batch, experimental week, day (factor with six levels: 1-6), time of the day (factor with 11 levels: 0:00, 2:00, 4:00, 6:00, 10:00, 12:00, 14:00, 16:00, 18:00, 20:00 and 22:00), pen unit, phase of the day (factor with two levels: light and dark) and their interactions as fixed effects. F-tests of overall significance $(p<0.05)$ were calculated in order to retain meaningful variables and interactions only, apart from the time of the day, which was inevitably kept. The final model was calculated for each experimental week (i.e., weeks 1,3 and 5) and included the day and time of the day only.

The MIXED procedure was also used to examine the "lying", "eating" and "active" behaviours of the piglets. Variance components were estimated using the restricted maximum likelihood (REML) method. For the residual effects, heterogeneous residual variances were modelled (grouped according to the pen unit and phase of the day). The models included the same fixed effects than the colour temperature preference model. F-tests of overall significance $(p<0.05)$ were calculated in order to retain meaningful variables and interactions only, apart from the compartment colour temperature and time of the day, which were inevitably kept. The final models were calculated for each experimental week (i.e., weeks 1, 3 and 5) and included the day, time of the day, compartment colour temperature and the time of the day $\times$ compartment colour temperature interaction. 


\section{Results}

\subsection{LED Light Colour Temperature Preference}

A total of 84,704 behavioural observations were recorded by using the time sampling method, including 50,943 observations (i.e., $60.43 \%$ of all the observations) made under a colour temperature of $3000 \mathrm{~K}$ and 33,761 observations (i.e., 39.57\%) made under $6500 \mathrm{~K}$ (Table 1). The "lying" behaviour was the most observed behaviour (i.e., $85.70 \%$ of all the observations), whereas the "eating" behaviour represents $6.16 \%$ and the "active" behaviour $8.14 \%$ of all the observations (Table 1 ).

Table 1. Distribution of the behaviours observed (in \%) under the $3000 \mathrm{~K}$ and $6500 \mathrm{~K}$ LED light colour temperatures.

\begin{tabular}{ccccc}
\hline \multirow{2}{*}{ Colour Temperature } & \multicolumn{4}{c}{ Observed Behaviours (\%) } \\
\cline { 2 - 5 } & "Lying" & “Eating" & "Active" & Total \\
\hline $3000 \mathrm{~K}$ & 53.50 & 2.71 & 4.22 & 60.43 \\
$6500 \mathrm{~K}$ & 32.20 & 3.45 & 3.92 & 39.57 \\
Total & 85.70 & 6.16 & 8.14 & \\
\hline
\end{tabular}

The animals showed a preference for the LED light colour temperature of $3000 \mathrm{~K}$ in the first experimental week throughout the day ( $p \leq 0.003$, except at 16:00 where $p=0.052$; Figure 3a), with on average $71.3 \%$ of the pigs staying under this colour temperature. In the third experimental week, this preference decreased with pigs favouring the LED light colour temperature of $3000 \mathrm{~K}$ only between 4:00 and 8:00 ( $p \leq 0.015)$ and between 20:00 and 0:00 ( $p \leq 0.024$; Figure 3b). In the fifth experimental week, no colour temperature preference was detected anymore ( $p=0.31$; Figure $3 c)$.

(a)
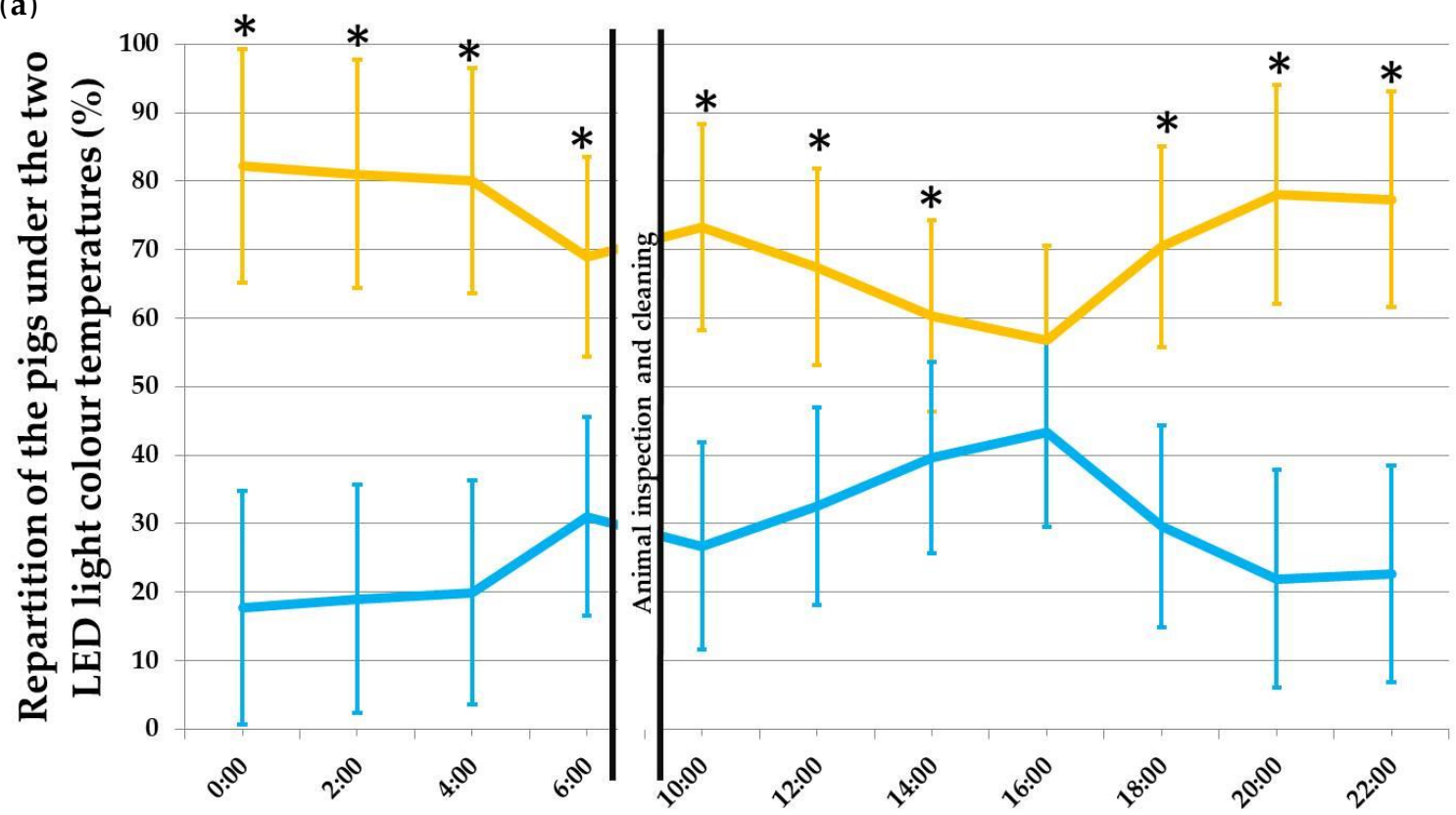

Time of the day (h)

Figure 3. Cont. 


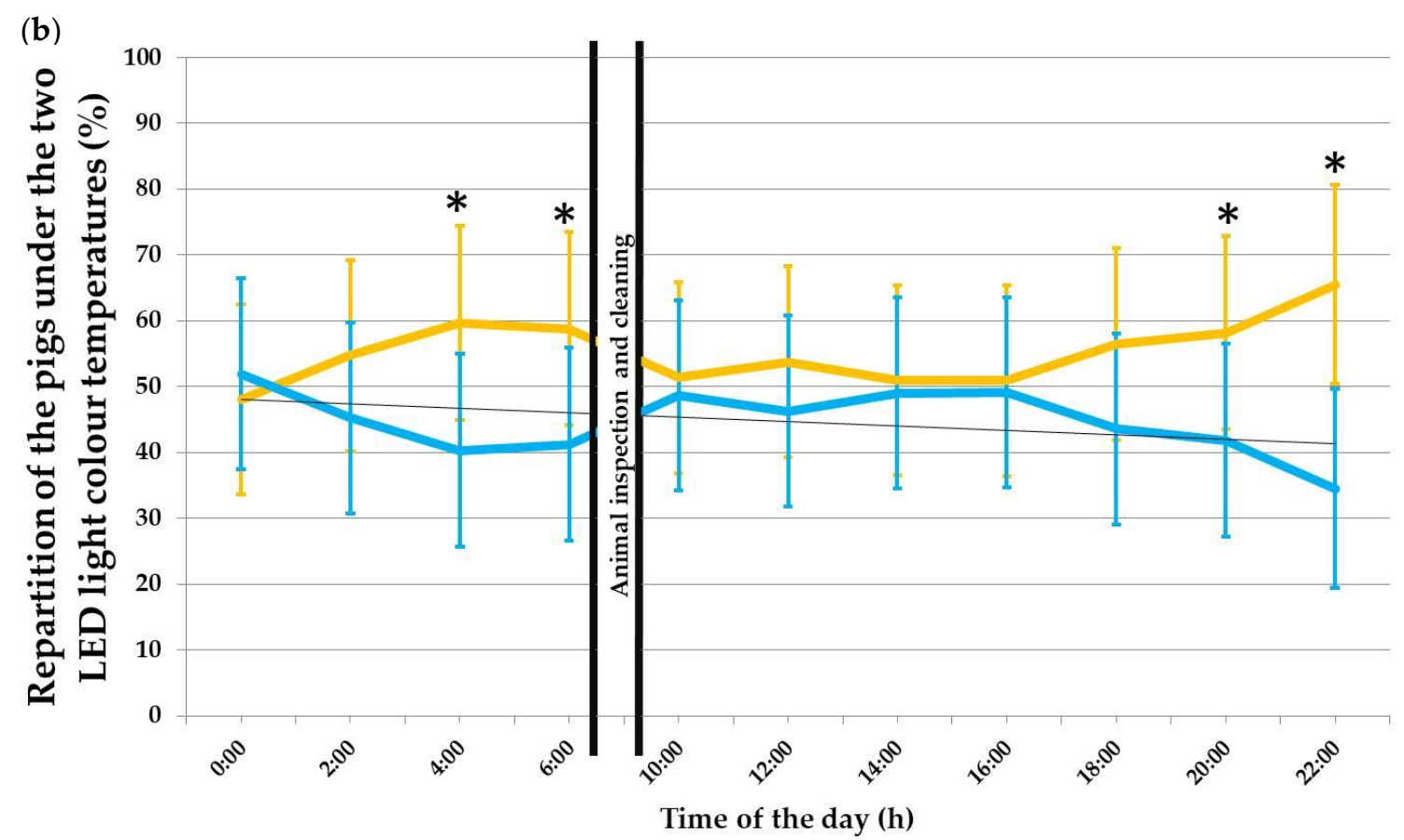

(c)

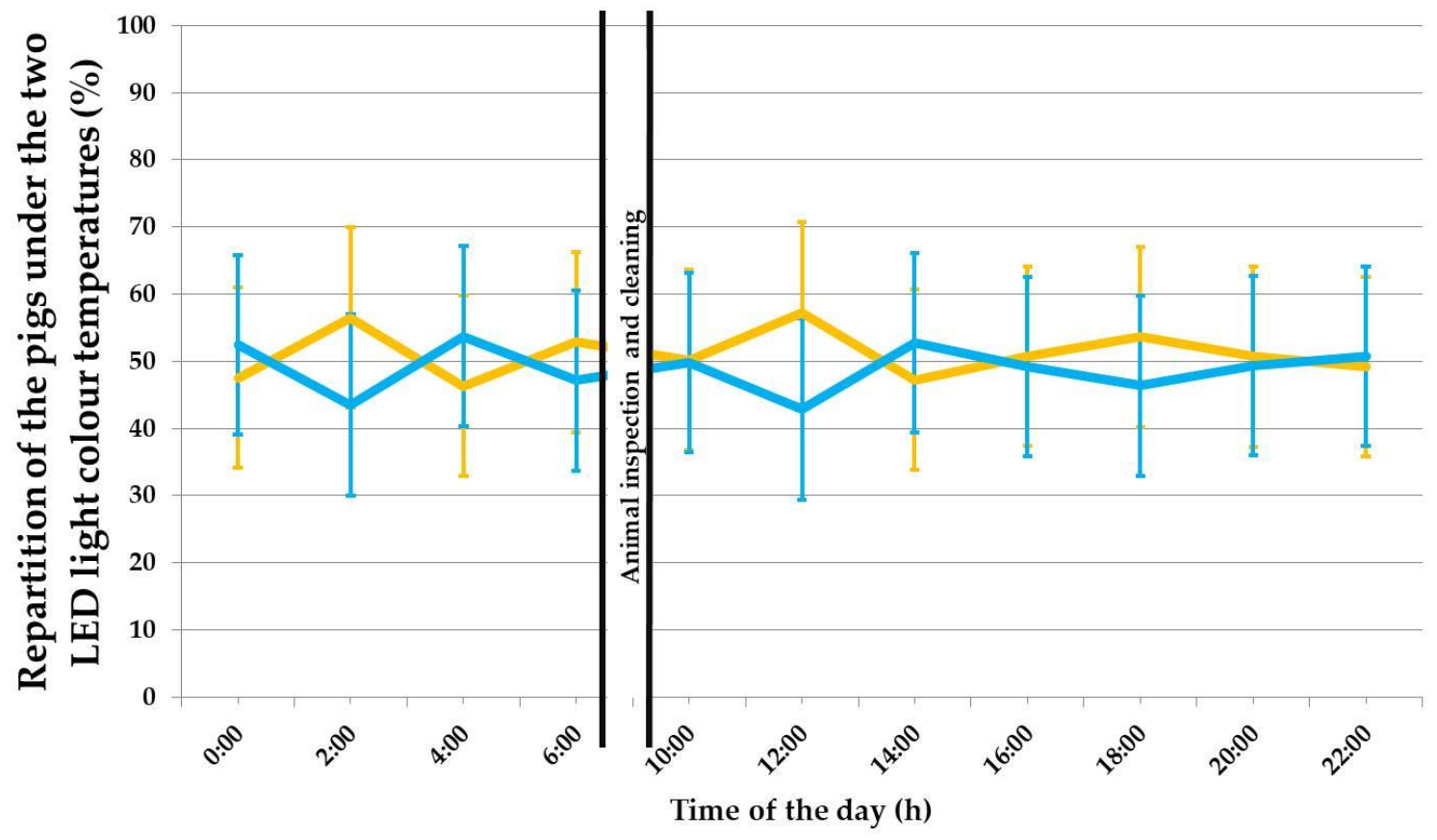

Figure 3. Repartition of the pigs (in \%) throughout the day $(24 \mathrm{~h})$ under the LED light colour temperatures of $3000 \mathrm{~K}$ (in yellow) and $6500 \mathrm{~K}$ (in blue) during (a) the first, (b) third and (c) fifth experimental week. The error bars represent the standard error $( \pm \mathrm{SE})$ of the mean. An asterisk $\left.{ }^{*}\right)$ shows a statistical difference with $p<0.05$.

An effect of the day on the LED light colour temperature preference was observed throughout the experimental weeks ( $p \leq 0.002$; Table 2). 
Table 2. Percentage of pigs observed for each recorded day (Days 1-3) of the first, third and fifth experimental week (with Batch 1 and 2 summed) under the $3000 \mathrm{~K}$ and $6500 \mathrm{~K}$ LED light colour temperatures.

\begin{tabular}{ccccccccccc}
\hline & \multicolumn{9}{c}{ Pigs Observed (\%) } \\
\cline { 2 - 11 } Colour Temperature & \multicolumn{3}{c}{ Week 1 } & \multicolumn{3}{c}{ Week 3 } & & Week 5 \\
\cline { 2 - 11 } & Day 1 & Day 2 & Day 3 & Day 1 & Day 2 & Day 3 & Day 1 & Day 2 & Day 3 \\
\hline 3000 Kelvin & 72.5 & 76.7 & 67.3 & 52.8 & 56.4 & 56.1 & 49 & 50.4 & 53.8 \\
6500 Kelvin & 27.5 & 23.3 & 32.7 & 47.2 & 43.6 & 43.9 & 51 & 49.6 & 46.2 \\
\hline
\end{tabular}

\section{2. "Lying" Behaviour}

The "lying" behaviour was the most performed behaviour with on average $85.70 \%$ of the pigs lying down (Figure 4). Throughout the experiment, the compartment colour temperature $(p<0.001)$, time of the day $(p \leq 0.0012)$ and their interaction $(p<0.004)$ had an effect on the "lying" behaviour, which was preferably performed under the colour temperature of $3000 \mathrm{~K}$. In the first experimental week, the time of the day $\times$ compartment colour temperature interaction highlighted a clear preference for $3000 \mathrm{~K}$ throughout the day (Figure $4 \mathrm{a}$ ). This preference then decreased across the weeks, with the colour temperature of $3000 \mathrm{~K}$ being preferred only from 2:00 to 8:00 ( $p \leq 0.036)$, at 12:00 ( $p \leq 0.022)$ and from 18:00 to 0:00 ( $p \leq 0.0014)$ in the third week (Figure $4 b$ ), and only at 2:00, 12:00 and 18:00 in the fifth week (Figure $4 \mathrm{c})$. The day $(p \geq 0.84)$ had no effect on pigs' "lying" behaviour.

(a)
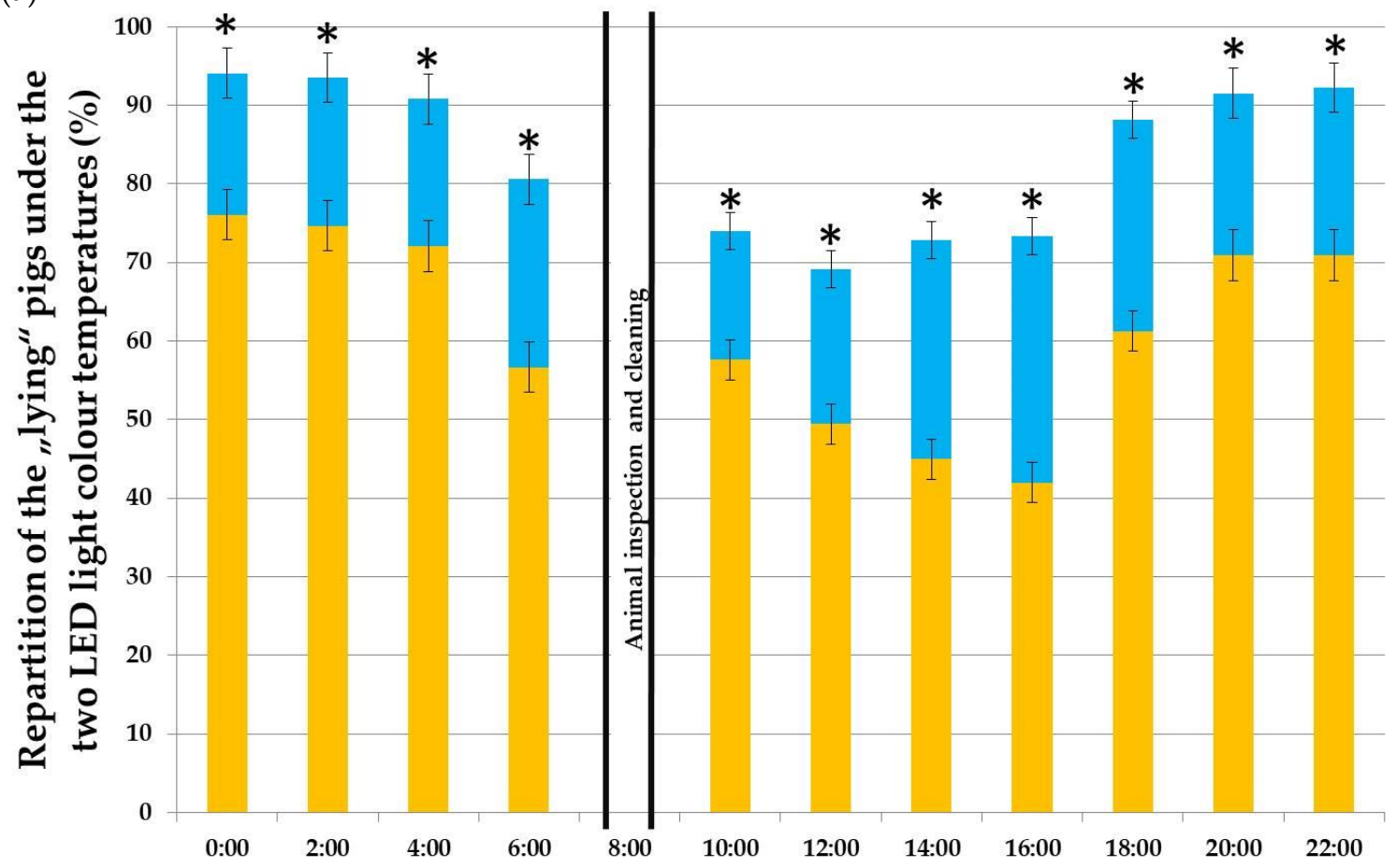

Time of the day $(\mathrm{h})$

Figure 4. Cont. 

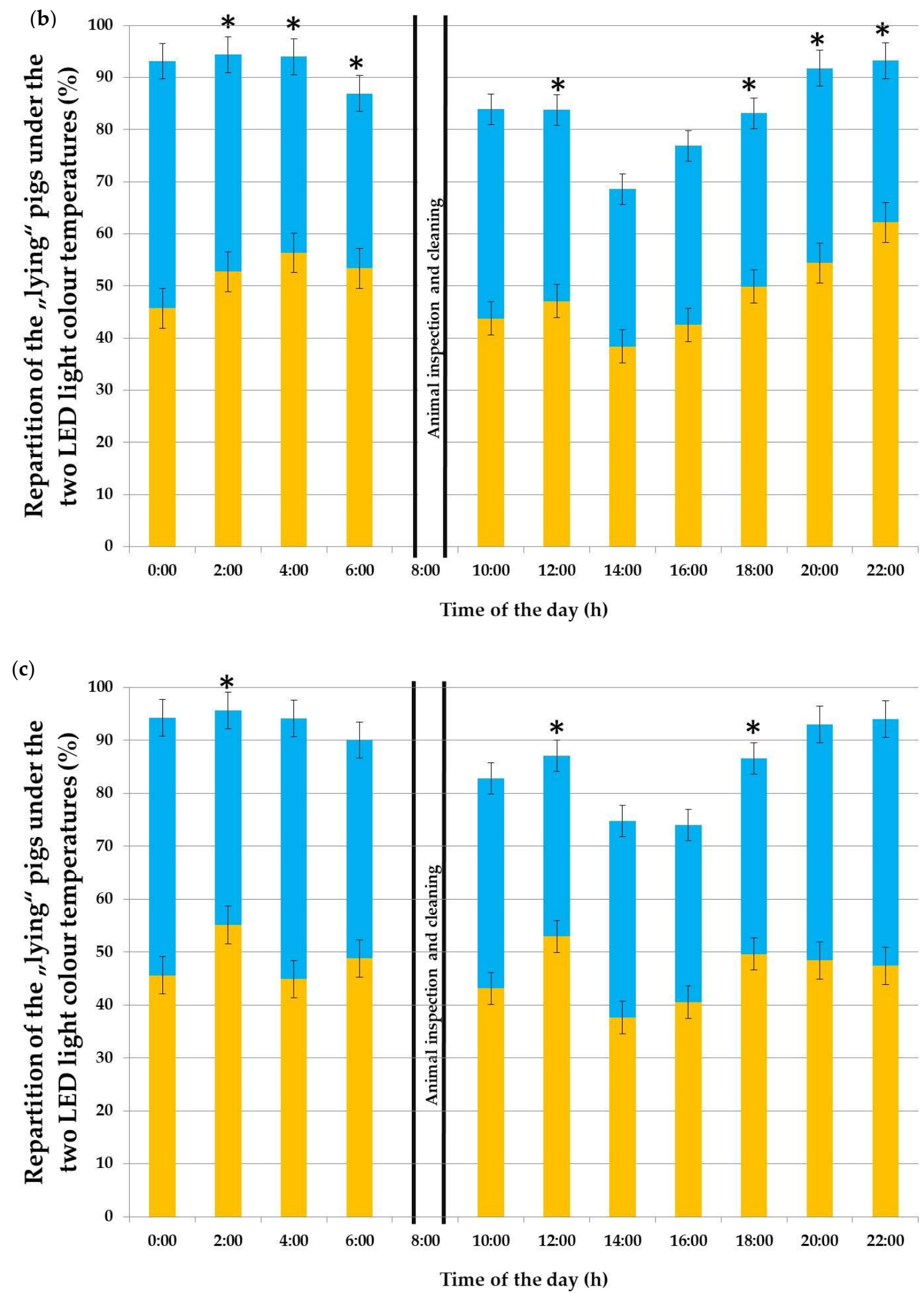

Figure 4. Repartition of the "lying" pigs (in \%) throughout the day (24 h) under the LED light colour temperatures of $3000 \mathrm{~K}$ (in yellow) and $6500 \mathrm{~K}$ (in blue), given in percentage of the total performed behaviours, during (a) the first, (b) third and (c) fifth experimental week. The error bars represent the standard error $( \pm \mathrm{SE})$ of the mean. An asterisk $\left(^{*}\right)$ shows a statistical difference in the time of the day $x$ compartment colour temperature interaction with $p<0.05$. 


\section{3. "Eating" Behaviour}

The "eating" behaviour was shown on average by $6.16 \%$ of the pigs at the time, mainly between 6:00 and 20:00.

In the first experimental week (Figure 5a), the compartment colour temperature $(p<0.001)$ and the time of the day $(p<0.001)$ had an effect on the "eating" behaviour, with a preference for eating under the colour temperature of $3000 \mathrm{~K}$. However, the colour temperature $\times$ time interaction $(p=0.34)$ and day $(p>0.98)$ had no effect.

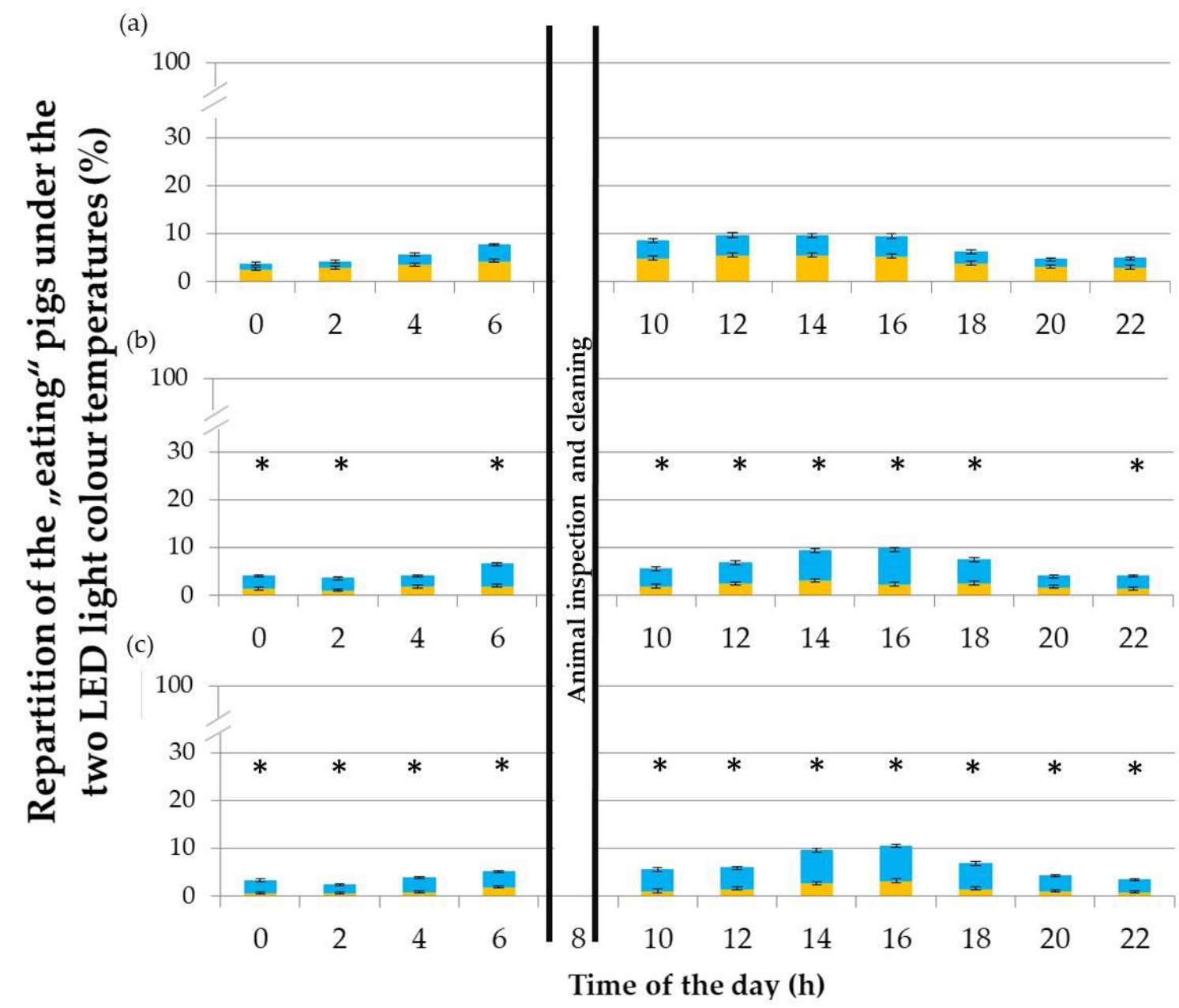

Figure 5. Repartition of the "eating" pigs (in \%) throughout the day ( $24 \mathrm{~h}$ ) under the LED light colour temperatures of $3000 \mathrm{~K}$ (in yellow) and $6500 \mathrm{~K}$ (in blue), given in percentage of the total performed behaviours, during (a) the first, (b) third and (c) fifth experimental week. The error bars represent the standard error $( \pm \mathrm{SE})$ of the mean. An asterisk $\left(^{*}\right)$ shows a statistical difference in the time of the day $\times$ compartment colour temperature interaction with $p<0.05$.

In the third experimental week (Figure 5b), the compartment colour temperature $(p<0.001)$, time of the day $(p<0.001)$ and their interaction $(p<0.001)$ had an effect on the "eating" behaviour, with a preference for the colour temperature of $6500 \mathrm{~K}$ throughout the day $(p \leq 0.026)$, except at 4:00 and 20:00 (where $p \geq 0.56$ ). However, the day $(p=0.53$ ) had no effect.

In the fifth experimental week (Figure $5 c)$, the compartment colour temperature $(p<0.001)$, time of the day $(p<0.001)$ and the colour temperature $\times$ time interaction $(p<0.001)$ had an effect on the "eating" behaviour, with a preference for the colour temperature of $6500 \mathrm{~K}$ throughout the day $(p \leq 0.032)$. 


\section{4. "Active" Behaviour}

The "active" behaviour was performed on average by $8.14 \%$ of the pigs, mostly between $6: 00$ and 18:00. However, no clear overall preference for one or the other LED light colour temperature was found.

In the first week (Figure 6a), the time of the day $(p<0.001)$, day $(p<0.001)$ and compartment colour temperature $(p<0.003)$ had an effect on the "active" behaviour, with a preference for the colour temperature of $3000 \mathrm{~K}$. The colour temperature $\times$ time interaction $(p=0.33)$ had no effect.

(a)
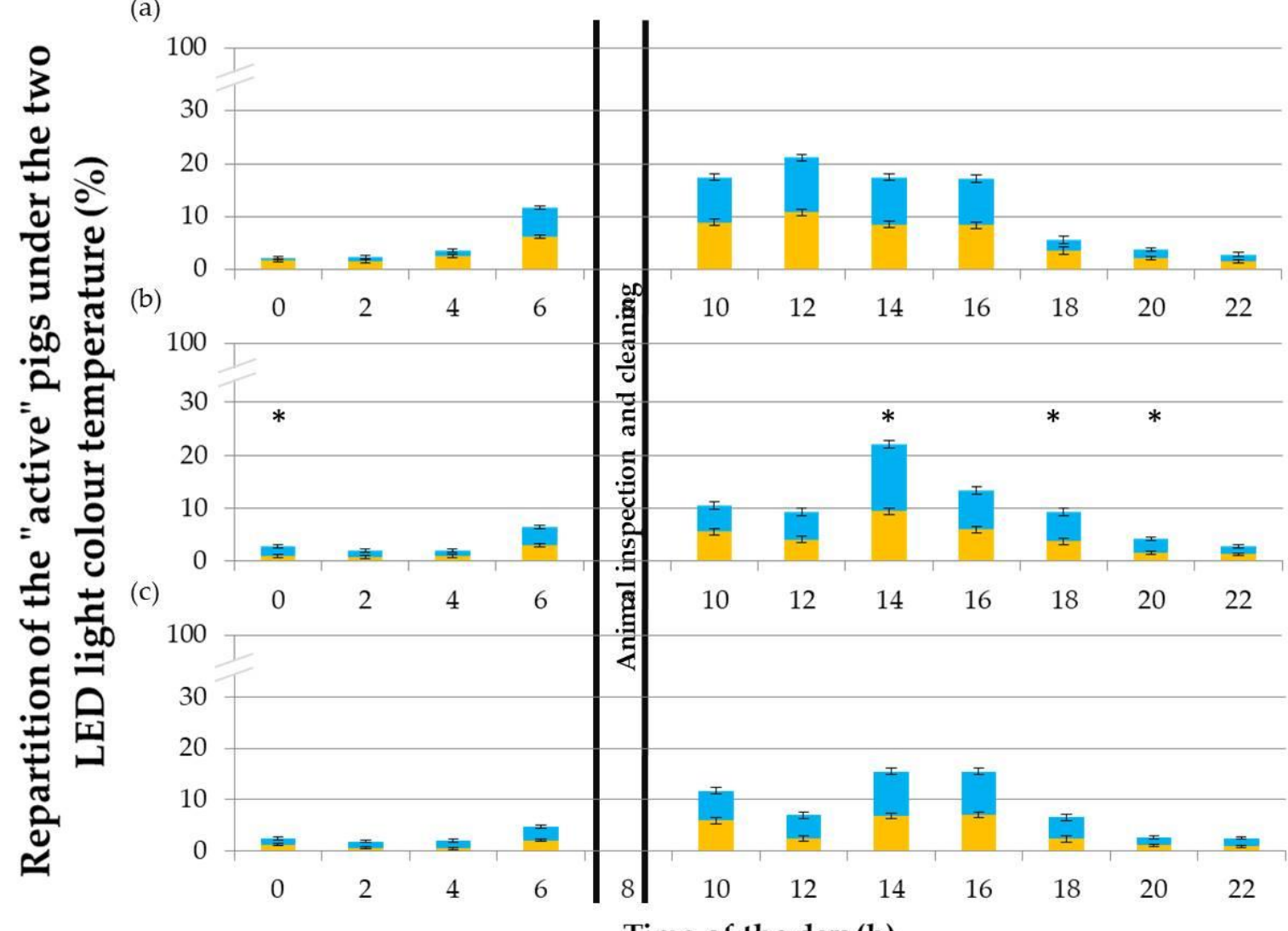

Time of the day (h)

Figure 6. Repartition of the "active" pigs (in \%) throughout the day (24 h) under the LED light colour temperatures of $3000 \mathrm{~K}$ (in yellow) and $6500 \mathrm{~K}$ (in blue), given in percentage of the total performed behaviours, during (a) the first, (b) third and (c) fifth experimental week. The error bars represent the standard error $( \pm \mathrm{SE})$ of the mean. An asterisk $(*)$ shows a statistical difference in the time of the day $\times$ compartment colour temperature interaction with $p<0.05$.

In the third week (Figure $6 \mathrm{~b}$ ), the time of the day $(p<0.001)$, compartment colour temperature $(p<0.001)$ and colour temperature $\times$ time interaction $(p=0.038)$ had an effect on the "active" behaviour, with a preference for the colour temperature of $6500 \mathrm{~K}$ at 0:00, 14:00, 18:00 and 20:00 ( $p \leq 0.041)$. The day $(p=0.13)$ had however no effect.

In the fifth week (Figure $6 c)$, the time of the day $(p<0.001)$, day $(p<0.001)$ and compartment colour temperature $(p<0.001)$ had an effect on the "active" behaviour, with a preference for the colour temperature of $6500 \mathrm{~K}$. However, the colour temperature $\times$ time interaction $(p=0.22)$ had no effect.

\subsection{Pen Compartment Cleanliness}

The LED light colour temperature was found to have an effect $\left(\chi^{2}{ }_{3}=58.32, p<0.001\right.$; Figure 7$)$ on the pen compartment cleanliness scores. Pen compartments illuminated with the colour temperature 
of $6500 \mathrm{~K}$ were more frequently given the scores 2 (lightly soiled) and 3 (moderately soiled) than pen compartments illuminated at $3000 \mathrm{~K}$ (Figure 7). In fact, the score 0 (not soiled) was only given in pen compartments under the colour temperature of $3000 \mathrm{~K}$.

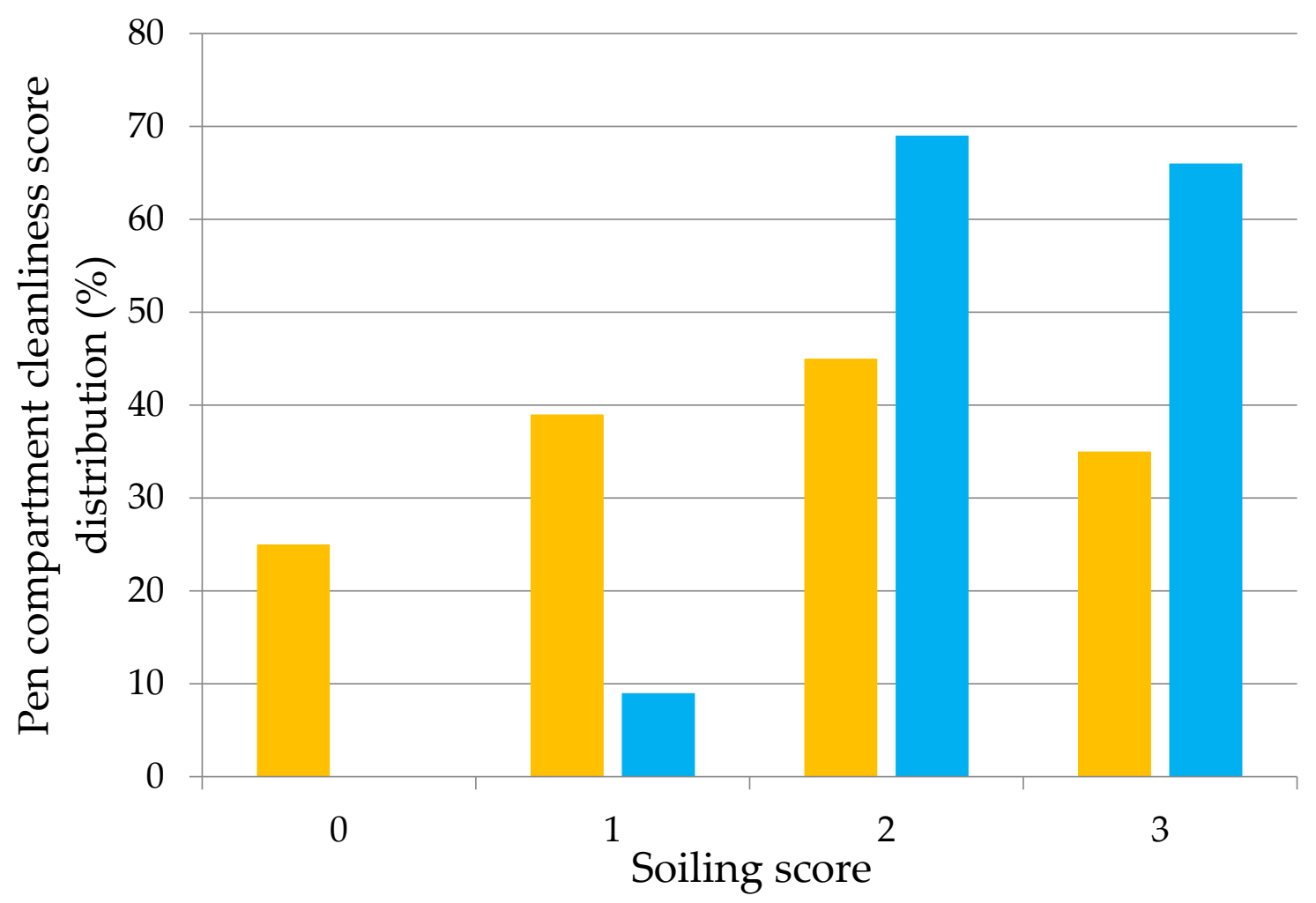

Figure 7. Pen compartment cleanliness score distribution (in \%) under $3000 \mathrm{~K}$ (in yellow) and $6500 \mathrm{~K}$ (in blue) LED light colour temperatures, with the following cleanliness scores: $0=$ not soiled; $1=$ very lightly soiled; 2 = lightly soiled; 3 = moderately soiled; and 4 = highly soiled.

\subsection{Feed Consumption}

Pigs ate $15.1 \pm 3.0 \mathrm{~kg}(\mathrm{LSM} \pm \mathrm{SE})$ of feed the first week, $23.9 \pm 5.7 \mathrm{~kg}$ the second week, $30.4 \pm 7.1 \mathrm{~kg}$ the third week, $37.7 \pm 11.1 \mathrm{~kg}$ the fourth week and $43.8 \pm 11.2 \mathrm{~kg}$ the fifth week.

The LED light colour temperature had an effect on the pigs' feed consumption $(p=0.046)$. Pigs consumed more feed under the colour temperature of $6500 \mathrm{~K}(38.0 \pm 5.2 \mathrm{~kg})$ than of $3000 \mathrm{~K}$ $(22.3 \pm 5.2 \mathrm{~kg})$. The experimental week $(p=0.067)$ and colour temperature $\times$ week interaction $(p=0.134)$ were found to have no effect on feed consumption.

\section{Discussion}

The aim of the study was to investigate whether piglets had a preference between the LED light colour temperatures of $3000 \mathrm{~K}$ (reddish for the human vision) and $6500 \mathrm{~K}$ (bluish). During 5 weeks, the percentage of pigs "lying", "eating" and being "active" was recorded in each colour temperature compartment. The amount of feed consumed as well as the pen compartment soiling were also evaluated for each colour temperature. In the first experimental week, a clear preference for the colour temperature of $3000 \mathrm{~K}$ was observed, under which all the measured behaviours were more frequently performed throughout the day. However, in the following weeks this colour temperature preference faded. On the other hand, feed consumption and pen compartment soiling were higher under the colour temperature of $6500 \mathrm{~K}$.

In our study, pigs showed a clear preference for the LED light colour temperature of $3000 \mathrm{~K}$ in the first experimental week, with on average more than $2 / 3$ of the pigs staying under this colour. However, this preference diminished in the third experimental week to appear only on the early morning and 
late evening hours, before completely fading in the fifth experimental week. Though it remains unclear why pigs had an initial preference for the colour temperature of $3000 \mathrm{~K}$ and no colour temperature preference anymore at the end of the experiment, this preference for "warmer" reddish light has also been observed in mice that were given the choice of being exposed to a colour temperature of $2500 \mathrm{~K}$ or $4000 \mathrm{~K}$ at 91 lux [13]. Kapogiannatou et al. [14] observed that mice were less stressed under a light colour temperature of $2500 \mathrm{~K}$ than one of $4000 \mathrm{~K}$ at $60 \mathrm{lux}$, while spontaneous behaviours and locomotor activity levels were unaffected. Accordingly, Oosterhout et al. [15] also related that the behavioural activity and biorhythm of dairy cows were not (or least in comparison to shorter wavelengths) affected by long wavelengths' (red) light.

Respectively, a preference for lying down under the colour temperature of $3000 \mathrm{~K}$ was observed for the entire test period. This preference diminished across the experimental weeks, appearing then at a certain time of the day only. These observations concurred with those of the global colour temperature preference. Indeed, the lying behaviour was the most exhibited behaviour, representing on average more than $4 / 5$ of the pigs. This is in agreement with Sambraus [18] and Marx [19], who found that conventionally housed pigs spent $80-90 \%$ of the day lying down. Moreover, a study of Hoy [20] previously examined the influence of different factors on the choice for resting place in pigs and revealed that the room temperature, texture of the ground and space available per pig are the most influencing factors. One explanation for the decrease in the light colour temperature preference with increasing weeks could therefore be the relative decrease of space availability due to the increasing size of the piglets that grew up (with $2.0 \mathrm{~kg}$ of live weight $/ \mathrm{m}^{2}$ in the first vs. $7.1 \mathrm{~kg}$ of live weight $/ \mathrm{m}^{2}$ in the fifth week). This might be checked by considerably increasing the minimal area per pig or by decreasing the density so that this factor would no longer be limiting.

Concerning the pen compartments' cleanliness, compartments providing a colour temperature of $6500 \mathrm{~K}$ were found to be more often and heavily soiled than those offering a colour temperature of $3000 \mathrm{~K}$. The most plausible explanation is that pigs avoid eliminating where they lie if the conditions allow it $[21,22]$. Indeed, as explained, pigs were observed to mainly lie down under the colour temperature of $3000 \mathrm{~K}$. It is thus not surprising to observe more excrements in the compartments offering a colour temperature of $6500 \mathrm{~K}$. In a recent study, Nannoni et al. [23] further explained that pigs probably first choose the most comfortable place for resting and then avoid eliminating there.

Overall, the eating behaviour represented the lowest percentage of all the behaviours recorded, and seemed to mainly take place during the light phase.

Štuhec et al. [24] also observed that conventionally housed pigs ate only $140 \mathrm{~min}$ per day, while Schiwitz [25] reported an even lower feeding duration in pigs (i.e., $28 \mathrm{~min}$ and $37 \mathrm{~min}$ out of $15 \mathrm{~h}$ observation on a fully slatted floor and in a bedding system, respectively). Although the pigs in the present study initially preferred to eat under the colour temperature of $3000 \mathrm{~K}$, this preference was quickly reversed (from the third experimental week onwards), with pigs thus eating more often under the colour temperature of $6500 \mathrm{~K}$. Regarding the feed consumption, we noticed that the pigs' consumption was overall higher under the colour temperature of $6500 \mathrm{~K}$. While rabbits' feed consumption does not seem to be affected by the light colour [26], broiler chickens were observed gaining more weight under a "colder" bluish light $[7,8]$. However, care should be given when comparing our results with those of chickens because they have very different vision (i.e., a tetrachromatic view).

Pigs were found to be mostly active during the light phase of the day, though this overall activity was found to represent only a small percentage of the whole behaviours performed that we recorded. Similar observations were made by Zaludik [27], who reported that pigs kept on partially or fully slatted floors showed an active behaviour only up to $8.8 \%$ of the time (as based on an observation period of $7 \mathrm{~h}$ ). However, for the active behaviour, no clear preference for one of the colour temperatures could be identified. Pigs that showed this active behaviour were often playing together and chasing each other. They would independently cross the pen compartments and therefore be recorded as active under both colour temperatures. Pigs were also frequently observed moving from one feeding trough to another when they were not given the opportunity to eat. Furthermore, the times of the day 
where the active behaviour was more frequently recorded coincide with the times of day where pigs were seen eating. This is probably due to the fact that pigs that tried to get to the feeding trough and displaced other pigs were also considered "active". The day effect on the active behaviour of the pigs observed during the first experimental week could result from a higher exploratory behaviour on the first day as piglets certainly actively explored their new light regiment environment.

Finally, a day effect could be noticed on the pigs' LED light colour temperature preference, as well as on their eating and active behaviour, but not on their lying behaviour. Throughout the experiment, attention was paid to keep the pen compartment conditions constant. Nevertheless, animals do not behave consistently from day to day. By selecting three different observation days per experimental week, we aimed to obtain a representative overview of the pigs' behaviour. Evaluating more days per week or using a different evaluation method could possibly reduce the effect of the day.

\section{Conclusions}

This study highlighted that pigs can discriminate between two LED light colour temperatures of $3000 \mathrm{~K}$ and $6500 \mathrm{~K}$ at an illuminance of 80 lux during the day. Colour temperature preference seems to vary according to the activity performed (e.g., eating, eliminative or resting behaviour) and across the weeks. Further investigations could focus on finding out whether the behaviour of pigs can be guided into specific functional areas by using different colour temperatures and/or brightness levels. This could enable the use of lighting to help structure the pens into clear functional areas. Due to the actual density in conventional pig housing, a clearer demarcation of the different areas could increase pig welfare and ease animal keepers' work.

Author Contributions: Conceptualization, E.v.B., D.W. and S.G.; methodology, S.G.; validation, C.M.C.R.; formal analysis, S.G. and M.W.-D.; investigation, S.G.; data curation, S.G., C.M.C.R. and M.W.-D.; writing-original draft preparation, S.G.; writing-review and editing, S.G., C.M.C.R., K.R., M.W.-D., D.W. and E.v.B.; visualization, S.G. and C.M.C.R.; supervision, K.R. and E.v.B.; project administration, S.G.; funding acquisition, K.R. All authors have read and agreed to the published version of the manuscript.

Funding: This study was carried out as part of the "Innovative LED luminaire for increased requirements in livestock farming" (InnoLED_4_Livestock). The project was supported by the German Government's Special Purpose Fund held at Landwirtschaftliche Rentenbank (Grant Nr. 820 204).

Acknowledgments: We would like to thank H. Nitzer and M. Zühl for their technical support during the experiments. Thanks also go to the Bielefeld University of Applied Sciences for its support concerning technical questions about light.

Conflicts of Interest: The authors declare no conflict of interest.

\section{References}

1. Hirt, A.; Maisack, C.; Moritz, J. Tierschutzgesetz: Mit TierSchHundeV, TierSchNutztV, TierSchVersV, TierSchTrV, EU-Tiertransport-VO, TierSchlV, EU-Tierschlacht-VO, 4th ed.; Vahlen, Franz: München, Germany, 2020.

2. European Union Council Directive. Council Directive 2008/120/EC of 18 December 2008 Laying Down Minimum Standards for the Protection of Pigs. Off. J. Eur. Union 2008, 316, 36-38.

3. NAWAC. Animal Welfare (Pigs) Code of Welfare 2010; Ministry of Agriculture and Forestry: Wellington, New Zealand, 2010.

4. The State of Victoria. Victorian Standards and Guidelines for the Welfare of Pigs; Department of Primary Industries, Biosecurity Victoria: Melbourne, VIC, Australia, 2012.

5. National Pork Board -Swine Care Handbook. Available online: https://egashops.directedje.com/PorkStoreP roducer/product-details.asp?ID=89\&CID=\&P=1 (accessed on 5 November 2020).

6. von Borell, E. Wieviel Licht braucht das Schwein? In Proceedings of the 21st International DVG Symposium on Animal Welfare, München, Germany, 25-27 February 2016; DVG: Gießen, Germany, 2016.

7. Archer, G.S. Color temperature of light-emitting diode lighting matters for optimum growth and welfare of broiler chickens. Animal 2018, 12, 1015-1021. [CrossRef] [PubMed]

8. Riber, A.B. Effects of color of light on preferences, performance, and welfare in broilers. Poult. Sci. 2015, 94, 1767-1775. [CrossRef] [PubMed] 
9. Tanida, H.; Senda, K.; Suzuki, S.; Tanaka, T.; Yoshimoto, T. Color discrimination in weanling pigs. Nihon Chikusan Gakkaiho 1991, 62, 1029-1034. [CrossRef]

10. Loeffler, K.; Gäbel, G. Anatomie und Physiologie der Haustiere, 13th ed.; UTB GmbH.; Ulmer: Stuttgart, Germany, 2013.

11. Frings, S.; Müller, F. Biologie der Sinne. Vom Molekül zur Wahrnehmung; Springer Spektrum: Berlin/Heidelberg, Germany, 2014.

12. Neitz, J.; Jacobs, G.H. Spectral sensitivity of cones in an ungulate. Vis. Neurosci. 1989, 2, 97-100. [CrossRef] [PubMed]

13. Paronis, E.; Kapogiannatou, A.; Paschidis, K.; Stasinopoulou, M.; Alexakos, P.; Skaliora, I.; Kostomitsopoulos, N.G. Lighting environment: What colour of light do male C57BL/6J prefer? Appl. Anim. Behav. Sci. 2018, 209, 99-103. [CrossRef]

14. Kapogiannatou, A.; Paronis, E.; Paschidis, K.; Polissidis, A.; Kostomitsopoulos, N.G. Effect of light colour temperature and intensity on the behaviour of male C57CL/6J mice. Appl. Anim. Behav. Sci. 2016, 184, 135-140. [CrossRef]

15. Van Oosterhout, F.; Biewenga, G.; Winkel, A.; Ouweltjes, W.; Meijer, J.H.; Van Oosterhout, F.; Biewenga, G.; Winkel, A.; Ouweltjes, W.; Meijer, J.H. The effect of light colour and photoperiod on milk production and behaviour of dairy cows. Wagening. UR Livest. Res. 2012, 652, 1-31.

16. Federal Institute for Occupational Safety and Health. Technische Regeln für Arbeitsstätten (ASR)-Beleuchtung; BAuA: Dortmund, Germany, 2011.

17. Birkenfeld, C.; Schopfer, U.; Jais, C.; Peschke, W. Buchtensauberkeit in Ställen Mit Teilspaltenboden Für Mastschweine; Bavarian State Office for Agriculture: Freising, Germany, 2007.

18. Sambraus, H.H. Nutztierkunde; Ulmer: Stuttgart, Germany, 1991.

19. Marx, D. Beurteilungskriterien für artgerechte Tierhaltung am Beispiel der Schweineaufzucht. Bau. Für Die Landwirtsch 1991, 28, 6-10.

20. Hoy, S. Zu den Anforderungen von Mastschweinen an die Buchtenfläche. Tierärztliche Umsch. 2004, 59, 576-582.

21. Mollet, P.; Wechsler, B. Auslösende Reize für das Koten und Harnen bei Schweinen. KTBL Schr. 1991, 344, 150-161.

22. Bogner, H.; Andreae, U. Verhalten Landwirtschaftlicher Nutztiere; Ulmer: Stuttgart, Germany, 1984.

23. Nannoni, E.; Aarnink, A.J.A.; Vermeer, H.M.; Reimert, I.; Fels, M.; Bracke, M.B.M. Soiling of pig pens: A review of eliminative behaviour. Animals 2020, 10, 2025. [CrossRef]

24. Štuhec, I.; Siard, N.; Meznaris, D. Verhaltensanomalien bei individuell gehaltenen Mastschweinen. KTBL Schr. 1992, 351, 77-86.

25. Schiwitz, I. Ethologischer Vergleich Verschiedener Haltungssysteme bei Mastschweinen. Doctoral Dissertation, University of Natural Resources and Applied Life Sciences, Vienna, Austria, 1990.

26. Gerencsér, Z.; Matics, Z.; Nagy, I.; Szendrő, E. Light colour preference of growing rabbits. Ital. J. Anim. Sci. 2009, 8, 205-207. [CrossRef]

27. Zaludik, K. Bewertung Praxisüblicher Mastschweinehaltungen in Nordrhein-Westfalen Hinsichtlich der Tiergerechtheit. Ph.D. Thesis, Hohenheim University, Stuttgart, Germany, 2002.

Publisher's Note: MDPI stays neutral with regard to jurisdictional claims in published maps and institutional affiliations.

(C) 2020 by the authors. Licensee MDPI, Basel, Switzerland. This article is an open access article distributed under the terms and conditions of the Creative Commons Attribution (CC BY) license (http://creativecommons.org/licenses/by/4.0/). 